\title{
Hardy Spaces on Compact Riemann Surfaces with Boundary
}

\section{Zuevsky $A^{*}$}

School of Mathematics, Statistics and Applied Mathematics, National University of Ireland, Galway, Ireland

\begin{abstract}
We consider the holomorphic unramified mapping of two arbitrary finite bordered Riemann surfaces. Extending the map to the doubles $X_{1}$ and $X_{2}$ of Riemann surfaces we define the vector bundle on the second double as a direct image of the vector bundle on first double. We choose line bundles of half-order differentials $\Delta_{1}$ and $\Delta_{2}$ so that the vector bundle $V_{\chi_{2}}^{X_{2}} \otimes \Delta_{2}$ on $X_{2}$ would be the direct image of the vector bundle $V_{\chi_{1}}^{X_{1}} \otimes \Delta_{2}$. We then show that the Hardy spaces $H_{2, J_{1}(p)}\left(S_{1}, V_{\chi_{1}} \otimes \Delta_{1}\right)$ and $H_{2, J_{2}(p)}\left(S_{2}, V_{\chi_{2}} \otimes \Delta_{2}\right)$ are isometrically isomorphic. Proving that we construct an explicit isometric isomorphism and a matrix representation $\chi_{2}$ of the fundamental group $\pi_{1}\left(X_{2}, p_{0}\right)$ given a matrix representation $\chi_{1}$ of the fundamental group $\pi_{1}\left(X_{1}, p_{0}^{\prime}\right)$. On the basis of the results of Alpay et al. and Theorem 3.1 proven in the present work we then conjecture that there exists a covariant functor from the category $\mathcal{R} \mathcal{H}$ of finite bordered surfaces with vector bundle and signature matrices to the category of Kreĭn spaces and isomorphisms which are ramified covering of Riemann surfaces.
\end{abstract}

Keywords: Half-order differentials; Hardy spaces; Riemann surfaces

\section{Introduction}

It is well known how to study Hardy spaces defined on a finite bordered Riemann surface [1-5]. For domains with more than one boundary component it is natural to introduce, besides the usual positive definite inner product on $H_{2}$, indefinite inner products. Those products may be introduced by picking up different signature matrices when integrating over different components of the boundary of the Riemann surface. In the paper Alpay et al. [1] a necessary and sufficient condition for such an indefinite inner product to be non-degenerate was obtained. It was shown that when this condition is satisfied one actually gets a Krein space. The result was obtained by using a covering map of the surface to the unit disk to construct an isomorphism to a Hardy-Kreı̆n space over the unit disk. Furthermore, each holomorphic mapping of the finite bordered Riemann surface onto the unit disk (which maps boundary to boundary) determines an explicit isometric isomorphism between this space and a usual vector-valued Hardy space on the unit disk with an indefinite inner product defined by an appropriate hermitian matrix. The mapping to the unit disk in Alpay et al. [1] serves as a tool to study of the Hardy-Krein space over the finite bordered Riemann surface which in turn has motivation from the point of view of the study of commuting tuples of non-self adjoint operators. As it is usual when studying Hardy spaces on a multiply connected domain, the elements of the space are sections of a vector bundle rather than functions. The main point of the paper by Alpay et al. [1] was to construct an appropriate extension of this bundle to the double of the finite bordered Riemann surface and to use Cauchy kernels for certain vector bundles on a compact Riemann surface. Hardy spaces on a finite bordered Riemann surface, including indefinite Hardy spaces, are important in the model theory for commuting non-self adjoint operators [6].

Half-order differentials play a very important role in the vertex opera-tor algebra approach to construction of partition and $n$-point functions for conformal field theories defined on Riemann surfaces [79]. In particular, the Szegö kernel [10] turned out to be key object in construction of correlation functions in free fermion conformal field theories/chiral algebras on a genus two Riemann surface sewed from two genus one Riemann surfaces [11].

In this work we replace a holomorphic mapping of a finite bordered
Riemann surface onto the unit disk by a holomorphic mapping of two arbitrary finite bordered Riemann surfaces $S_{1}$ and $S_{2}$, which we assume however to be unramified. In the spirit of Alpay et al. [1,12] one can introduce the extension of the vector bundles on a finite bordered Riemann surfaces to the respective doubles. Extending the map $F$ to the doubles $X_{1}$ and $X_{2}$ of Riemann surfaces $S_{1}$ and $S_{2}$ we define the vector bundle $V_{\chi_{2}}^{X_{2}}$ on $X_{2}$ as a direct image of the vector bundle $V_{\chi_{1}}^{X_{1}}$ over $X_{1}$. We choose line bundles of half-order differentials (i.e., square roots of the canonical bundles $\left.K_{x_{i}}, i=1,2\right) \Delta_{1}$ and $\Delta_{2}$ so that the vector bundle $V_{\chi_{2}}^{X_{2}} \otimes \Delta_{2}$ on $X_{2}$ would be the direct image of the vector bundle $V_{\chi_{1}}^{X_{1}} \otimes \Delta_{1}$. We then show that the Hardy spaces $H_{2, J_{1}(p)}\left(S_{1}, V_{\chi_{1}} \otimes \Delta_{1}\right)$ bundle and $H_{2, J_{2}(p)}\left(S_{2}, V_{\chi_{2}} \otimes \Delta_{2}\right)$ are isometrically isomorphic. Proving that we construct a.) an explicit isometric isomorphism; b.) a matrix representation $\chi_{2}$ of the fundamental group $\pi_{1}\left(X_{2}, p_{0}\right)$ given a matrix representation $\chi_{1}$ of the fundamental group $\pi_{1}\left(X_{1}, p_{0}^{\prime}\right)$. Using results of Alpay et al. [1] and Theorem 3.1 proven in the present work we then conjecture that there exists a covariant functor from the category $\mathcal{R \mathcal { H }}$ of finite bordered surfaces with vector bundle and signature matrices to the category of Krein spaces and isomorphisms which are ramified covering of Riemann surfaces.

The isomorphism established in this work has also an operator theoretical interpretation, namely, a (ramified) covering $F$ allows us to construct a pair of commuting non-self adjoint operators with the model space on $S_{2}$ given a pair of commuting non-self adjoint operators with the model space on $S_{1}$. More generally, one might expect also possible connections with vessels construction and Bezoutians [13].

${ }^{*}$ Corresponding author: Zuevsky A, School of Mathematics, Statistics and Applied Mathematics, National University of Ireland, Galway, Ireland, Tel: 3531439 2424; E-mail: zuevsky@mpim-bonn.mpg.de

Received July 21, 2015; Accepted August 02, 2015; Published August 31, 2015

Citation: Zuevsky A (2015) Hardy Spaces on Compact Riemann Surfaces with Boundary. J Generalized Lie Theory Appl S1: 005. doi:10.4172/1736-4337.S1-005

Copyright: ๑ 2015 Zuevsky A. This is an open-access article distributed under the terms of the Creative Commons Attribution License, which permits unrestricted use, distribution, and reproduction in any medium, provided the original author and source are credited. 


\section{Preliminaries}

As we mentioned in Introduction indefinite Hardy spaces [14-16] on a finite bordered Riemann surface were considered in Alpay et al. [1].

Definition. Let $J$ be an $m \times m$ unitary self-adjoint matrix. Such a matrix is usually called a signature matrix. (In fact one may take $J$ to be any non-singular self-adjoint matrix). The Hardy space $H_{2}^{m}$ on the unit disk $\mathbf{D}$ endowed with the indefinite inner product

$$
[f, g]_{J}=\frac{1}{2 \pi} \int_{0}^{2 \pi} g\left(e^{i t}\right)^{*} J f\left(e^{i t}\right) d t
$$

is a Krĕn space denoted $H_{2}^{m}, J$.

This space plays an important role in interpolation theory [17] and in model theory [18]. For the general theory of Kreĭn spaces[19-21].

Suppose now that we have an open Riemann surface $S$ such that $S \cup \partial S$ is a finite bordered Riemann surface (i.e., a compact Riemann surface with boundary), with the boundary $\partial S$ consisting of $k \geq 1$ components $\mathcal{X}_{0}, \ldots, \mathcal{X}_{k-1}$. We consider analytic sections of a rank $m$ flat unitary vector bundle $V_{\chi}$ on $S$ corresponding to a homomorphism $\chi$ from the fundamental group $\pi_{1}\left(S, p_{0}\right)$ into the group $U(m)$ of $m \times m$ unitary matrices. An analytic section $f$ of $V_{x}$ over $S$ is an analytic $\mathbb{C}^{m_{-}}$ valued function on the universal covering $\tilde{S}$ of $S$ satisfying

$$
f(T \tilde{p})=\chi(T) f(\tilde{p}),
$$

for all $\tilde{p} \in \tilde{S}$ and all deck transformations $T$ of $\tilde{S}$ over $S$, which we identify with elements of the fundamental group $\pi_{1}(S, p) ; f$ can be thought of as a multiplicative multivalued function on $S$. We consider also multiplicative half-order differentials [1], i.e., sections of a vector bundle of the form $V_{\chi} \otimes \Delta$, where $V_{\chi}$ is a flat unitary vector bundle on $S$ as above and $\Delta$ is a square root of the canonical bundle on $S: \Delta \otimes \Delta$ $\cong K_{\mathrm{s}}$

Definition. The Hardy space $H_{2}(S, V \otimes \Delta)$ on a Riemann surface $S$ is the set of sections $\hat{f}$ of a vector bundle $V_{\chi} \otimes \Delta$ analytic in $S$ satisfying

$$
\sup _{1-\epsilon<r<1} \sum_{i=0}^{k-1} \int_{X_{i}(r)} \hat{f}(p)^{*} \hat{f}(p)<\infty,
$$

for some $\epsilon>0$. In (1) $\mathcal{X}_{i}(r)$ denotes smooth simple closed curves in $S$ approximating $\mathcal{X}_{i}, i=0, \ldots, k-1$ ([1]): if $z_{i}$ is a boundary uniformizer near the boundary component $\mathcal{X}_{i}$ then $\mathcal{X}_{i}(r)$ is given by $\left|z_{i}(p)\right|=r$.

Note that since $\hat{f}(p)$ is a section of $V_{x} \otimes \Delta$, the expression $\hat{f}(p)^{*} \hat{f}(p)$ is a section of $\left|K_{s}\right|$, where $\left|K_{s}\right|^{\chi}$ is the line bundle with transition functions the absolute values of the transition functions of $K_{s}$; sections of $\left|K_{s}\right|$ can be represented locally as $\eta(t)|d t(p)|$ where $t(p)$ is a local parameter. Therefore one can integrate $\hat{f}(p)^{*} \hat{f}(p)$ over curves in $S$ and (1) makes sense.

The space $H_{2}\left(S, V_{x} \otimes \Delta\right)$ is a Hilbert space with the inner product

$$
\langle\hat{f}, \hat{g}\rangle=\lim _{r \rightarrow 1} \sum_{i=0}^{k-1} \int_{\mathcal{X}_{i}(r)} \hat{g}(p)^{*} \hat{f}(p) .
$$

For a relation between $H_{2}\left(S, V_{\chi} \otimes \Delta\right)$ and Hardy spaces of functions on $S$ with respect to a harmonic measure on $\partial S$, see Alpay et al. [1].

Definition. Denote by $H_{2, J(p)}\left(S, V_{x} \otimes \Delta\right)$ the analogue of the Krein space $H_{2, j}^{m}$ for $S$ which is the Hardy space $H_{2}\left(S, V_{\chi} \otimes \Delta\right)$ endowed with the indefinite inner product

$$
[\hat{f}, \hat{g}]_{J(p)}=\sum_{i=0}^{k-1} \int_{\mathcal{X}_{i}} \hat{g}(p)^{*} J(p) \hat{f}(p),
$$

where $\hat{f}(p)$ is the non-tangential boundary values of $\hat{f}(p)$ which exists again almost everywhere on $\partial S$ (Alpay et al.[1]) and $J(\tilde{p})$ is a locally constant matrix function on $\partial \tilde{S}$ whose values are $m \mathrm{x} m$ signature matrices, satisfying

$$
\chi(T)^{*} J(T \tilde{p}) \chi(T)=J(\tilde{p}),
$$

for all $\tilde{p} \in \partial \tilde{S}$ and all $T \in \pi_{1}\left(S, p_{0}\right)$. The expression $\hat{g}(p)^{*} J(p) \hat{f}(p)$ in (2) means $\hat{g}(\tilde{p})^{*} J(\tilde{p}) \hat{f}(\tilde{p})$ where $\tilde{p} \in \partial \tilde{S}$ is over $p \in \partial S$. It is a welldefined section of $\left|K_{s}\right|$ because of the transformation property of $J(\tilde{p})$. There exists certain freedom in the choice of $J(\tilde{p})$ for the given $V_{\gamma}$. Indeed, choose points $p_{i} \in \mathcal{X}_{i}, i=0, \ldots, k-1$. Let $C_{i}$ be a path on $S$ linking $p_{0}$ to $p_{i}$. Set $A_{i}=C_{i}^{-1} \mathcal{X}_{i} C_{i} \in \pi_{1}\left(S, p_{0}\right)$ (see Appendix). Then the (homotopy class of) $C_{i}$ determines a component $\tilde{\mathcal{X}}_{i}$ of $\partial \tilde{S}$ lying over $\mathcal{X}_{i}$, and the constant value $J_{i}$ of $J(\tilde{p})$ on $\tilde{\mathcal{X}}_{i}$ can be an arbitrary $m \mathrm{x} m$ signature matrix satisfying

$$
\chi\left(A_{i}\right)^{*} J_{i} \chi\left(A_{i}\right)=J_{i}
$$

Any other component of $\partial \tilde{S}$ lying over $\mathcal{X}_{i}$ can be obtained from $\tilde{\mathcal{X}}_{i}$ by some deck transformation $R$. The value of $J(\tilde{p})$ on this component is $\chi(R)^{*} J_{i} \chi(R)$. For the case of the line bundles (i.e., $m=$ $1)$, the choice of $J(\tilde{p})$ amounts to an arbitrary choice of a sign \pm 1 for each $\mathcal{X}_{i}$. We will often assume the choice of components $\tilde{\mathcal{X}}_{i}$ has been made and denote $H_{2, J(p)}\left(S, V_{\chi} \otimes \Delta\right)$ by $H_{2, J_{0}, \ldots, J_{k-1}}\left(S, V_{\chi} \otimes \Delta\right)$. The space $H_{2, J_{0}, \ldots, J_{k-1}}\left(S, V_{\chi} \otimes \Delta\right)$ is a natural example of an indefinite inner product space. It is related to the model theory of pairs of commuting non-selfadjoint operators and interpolation theory on multiply connected domains.

In the paper Alpay et al. [1] an appropriate extension of $V_{\chi}$ on $S$ to the double $X$ of the Riemann surface $S$ was constructed. Given a flat unitary vector bundle on a finite bordered Riemann surface, together with a collection of signature matrices, it can be uniquely extended to a flat unitary vector bundle on the double satisfying certain symmetry properties. Let us recall that construction.

Due to the identification of the boundaries the complex structures on two copies of $S$ constituting $X$ are mirror images of each other, i.e., there exists an anti-holomorphic involution $\tau: X \rightarrow X$ that maps $\bar{S}$ to $\bar{S}^{\prime}$. Thus $X$ is a compact real Riemann surface, or equivalently Riemann surface of a real algebraic curve. The genus $g$ of the double of $X$ of $S$ is $g$ $=2 s+k-1$, where $s$ is the genus of $S$. The set $X_{f}$ of fixed points of $\tau$ (real points of $X)$ coincides with the boundary $\partial S$ of $S$. Furthermore $X$ is a real Riemann surface of dividing type: the complement $X \backslash X_{f}$ consists of two connected components $X_{+}=S$ and $X_{-}=S^{\prime}$ interchanged by $\tau$. The converse is also true: any real Riemann surface of dividing type is the double of a finite bordered Riemann surface. The anti-holomorphic involution $\tau$ acts both on the fundamental group $\pi_{1}\left(X, p_{0}\right)$ and on the universal covering $\tilde{X}$ of $X$ (recall that the fundamental group $\pi_{1}\left(X, p_{0}\right)$ is isomorphic to the group of deck transformations Deck $(\tilde{X} / X)$. It also acts naturally on complex holomorphic vector bundles on $X$ : the transition functions for the vector bundle $V^{\tau}$ complex conjugates of the transition functions for $V$ at the point conjugate under $\tau$.

Consider a vector bundle $H$ on $X$ of rank $m$ with deg $H=m(g-1)$ satisfying the condition $h^{0}(H)=0$. Such a vector bundle is necessarily of the form $H \cong V_{\chi} \otimes \Delta$ where $V_{\chi}$ is a rank $m$ flat vector bundle on $X$ and $\Delta$ is a square root of $K_{\mathrm{x}}[22]$. These vector bundles are closely related to determinantal representations of algebraic curves and play an 
important role in the theory of commuting non-self adjoint operators and related theory of 2D systems [22-26].

Let $H$ be such that there exists a non-degenerate bilinear pairing $H$ $\times H^{\tau} \rightarrow K_{\mathrm{x}}$ which is parahermitian. The parahermitian property means that

$$
\left(\hat{f}, \hat{g}^{\tau}\right)(p)=\overline{\left(\hat{g}, \widehat{f^{\tau}}\right)\left(p^{\tau}\right)},
$$

for all local holomorphic sections $\hat{f}$ and $\hat{g}$ of $H$ near $p$ and $p^{\tau}$ respectively. We assume also that the line bundle $\Delta$ has been chosen so that $\Delta \cong \Delta^{\tau}$ and that the transition functions of $\Delta$ are symmetric with respect to $\tau$ [27]; Then

we obtain a parahermitian non-degenerate bilinear pairing $V_{\chi} \otimes V_{\chi}^{\tau} \rightarrow O_{X}$, or more explicitly an everywhere nonsingular holomorphic $m \times m$ matrix-valued function $G$ on the universal covering $\tilde{X}$ with the property

$$
G\left(\tilde{p}^{\tau}\right)^{*}=G(\tilde{p})
$$

satisfying the relation

$$
\chi\left(T^{\tau}\right)^{*} G(T \tilde{p}) \chi(T)=G(\tilde{p}),
$$

where $T \in \pi_{1}\left(X, p_{0}\right)$. The pairing $H \times H^{\tau} \rightarrow K_{\mathrm{X}}$ is then given explicitly by

$$
(\hat{f}, \hat{g})(\tilde{p})=\hat{g}\left(\tilde{p}^{\tau}\right)^{*} G(\tilde{p}) \hat{f}(\tilde{p}) .
$$

Now let us introduce the (in general) indefinite inner product

$$
[\hat{f}, \hat{g}]_{G(p)}=\int_{X_{f}} \hat{g}\left(\tilde{p}^{\tau}\right)^{*} G(\tilde{p}) \hat{f}(\tilde{p})
$$

where $\hat{f}$ and $\hat{g}$ are measurable sections of $H$ over $X_{f}$. Here and in similar expressions, the integral is computed on $X$ and the integrand does not depend on the choice of $\tilde{p} \in \tilde{X}$ above $p \in X$ Since in (6) $\tilde{p} \in \tilde{X}$ lies over a point of $X_{f}$ there exists $T_{\tilde{p}} \in \pi_{1}\left(X, p_{0}\right)$ such that $\tilde{p}^{\tau}=T_{\tilde{p}} \tilde{p}$. Therefore (6) can be rewritten as

$$
[\hat{f}, \hat{g}]_{G(p)}=\int_{X_{f}} \hat{g}(\tilde{p})^{*} J(\tilde{p}) \hat{f}(\tilde{p}),
$$

Where

$$
\begin{aligned}
& J(\tilde{p})=\chi\left(T_{\tilde{p}}\right)^{*} G(\tilde{p}) . \\
& \text { Note that } J(\tilde{p})^{*}=J(\tilde{p}) \text { and } \\
& \chi(R)^{*} J(R \tilde{p}) \chi(R)=J(\tilde{p}),
\end{aligned}
$$

for all $\tilde{p} \in \tilde{X}$ over $X_{f}$ and all $R \in \pi_{1}\left(X, p_{0}\right)$. Thus the vector bundle $H=$ $V_{\chi} \otimes \Delta$ on $X$ defines an indefinite inner product on the sections of its restriction to $X_{f}=\partial S$.

For $\tilde{p}$ lying over a point of $\mathcal{X}_{i}$, we have $T_{\tilde{p}}=R^{\tau} R^{-1}$ for $i=0$ and $T_{\tilde{p}}=R^{\tau} B_{i} R^{-1}$ for $i=1, \ldots, k-1$ where $B_{i}=\left(C_{i}^{\tau}\right)^{-1} C_{i}$ are part of the generators of the fundamental group $\pi_{1}\left(X, p_{0}\right)$ of $X$ (refer Appendix for the relation between generators of the fundamental groups of a Riemann surface $S$ and the corresponding double), and $R$ depends only on the component of the inverse image of $\mathcal{X}_{i}$ in $\tilde{X}$ that $\tilde{p}$ belongs to. Restricting $\tilde{p}$ in (7) to belong to a specific component we may write

$$
\begin{aligned}
& J(\tilde{p})=G(\tilde{p}) \\
& \text { for } i=0 \text { and } \\
& J(\tilde{p})=\chi\left(\mathrm{B}_{i}\right)^{*} G(\tilde{p}),
\end{aligned}
$$

for $i=1, \ldots, k-1$ (refer Appendix). (The specific component depends on the choice of the generators $B_{i}$, i.e., on the homotopy classes of the paths $C_{i}$.)

It follows from the conditions $\operatorname{deg} H=m(g-1)$ and $h^{0}(H)=0$ that $H$ is a semi-stable vector bundle. By a theorem of Narasimhan and Seshadri [28] $H$ is a direct sum of stable bundles if and only if the flat vector bundle $V_{x}$ (in $H=V_{x} \otimes \Delta$ ) can be taken to be unitary flat. Since $G$ is an isomorphism from ${ }^{\chi} V_{\chi}$ to the dual of $V_{x}^{\tau}$ it follows in this case that $G$ is constant and unitary. Since it is also selfadjoint, it is a constant signature matrix. Thus for analytic sections $\hat{g}$ and $\hat{f}$ of $V_{\chi} \otimes \Delta$ on $S$ that belong to $H_{2}\left(S, V_{\chi} \otimes \Delta\right)$ we can rewrite the inner product (7) as

$$
\begin{aligned}
{[\hat{f}, \hat{g}]_{G(p)} } & =\sum_{i=0}^{k-1} \int_{\chi_{i}} \hat{g}(\tilde{p})^{*} J_{i} \hat{f}(\tilde{p}) \\
& =[\hat{f}, \hat{g}]_{H_{2, J_{0}, \ldots, J_{k-1}}\left(S, V_{\chi} \otimes \Delta\right)},
\end{aligned}
$$

where

$$
J_{0}=G, J_{i}=\chi\left(B_{i}\right) * G
$$

for $i=1, \ldots, k-1$ and $p$ is restricted to belong to a specific component of the inverse image of $\mathcal{X}_{i}$ in $\tilde{X}$ as explained above. We then obtain for the vector bundle $H$ on $X$ the inner product (2) on the Hardy space $H_{2}, J_{0}, \ldots, J_{k-1}\left(S, V_{x} \otimes \Delta\right)$. Conversely, every unitary flat vector bundle on $S$ with signature matrices $J_{0}, \ldots, J_{k-1}$ can be obtained from a vector bundle $H$ on $X$ as above. Let $J_{i}, i=0, \ldots, k-1$ be self adjoint matrices and let $\chi: \pi_{1}\left(S, p_{0}\right) \rightarrow \mathrm{GL}(m, \mathbb{C})$ be a homomorphism satisfying

$$
\chi\left(A_{i}\right)^{*} J_{i} \chi\left(A_{i}\right)=J_{i} \text {. }
$$

Then by Proposition 2.1 from Alpay et al. [1] there exists a unique extension (still denoted by $\chi$ ) of $\chi$ to a homomorphism from $\pi_{1}\left(X, p_{0}\right)$ into $\mathrm{GL}(m, \mathbb{C})$ satisfying

$$
\begin{gathered}
\chi\left(T^{\tau}\right)^{*} G \chi(T)=G, \quad T \in \pi_{1}(X), \\
\chi\left(B_{i}\right)^{*} G=J_{i}
\end{gathered}
$$

where $G=J_{0}$ (refer Appendix).

If the original flat vector bundle $V_{\chi}$ on $S$ is unitary flat and all the matrices $J_{i}$ are unitary then the extended vector bundle is also unitary flat as it is follows form the proof of Proposition 2.1 of Alpay et al. [1]. The extension need not satisfy $h^{0}\left(X, V_{\chi} \otimes \Delta\right)=0$; i.e., the unitary case, this condition will be satisfied "generically" since flat unitary vector bundles $V$ on $X$ with $h^{0}(X, V, \otimes \Delta)>0$ form a divisor in the moduli space of flat unitary vector bundles (the generalized theta divisor $[10,29])$. It was proven in Proposition 2.2 from Alpay et al. [1] that if the indefinite inner product space $H_{2}, J_{0}, \ldots, J_{\mathrm{k}-1}\left(S, V_{x} \otimes \Delta\right)$ is nondegenerate, then

$$
h^{0}\left(X, V_{\chi} \otimes \Delta\right)=0 \text {. }
$$

It follows that the condition (10) is satisfied automatically in the positive definite case (i.e., when $J_{i}>0$ for $i=0, \ldots, k-1$ ); for line bundles, this has been obtained in Fay et al. [27,30].

Summing up, we see that the above extension procedure establishes a one-to-one correspondence between unitary flat vector bundles on $S$ together with a choice of signature matrices satisfying (3) and unitary flat vector bundle on $X$ satisfying the symmetry condition (4), (5). Given a unitary flat vector bundle on $S$, the various choices of extension to the double $X$ correspond to the various choices of signature matrices. We shall occasionally denote the corresponding unitary flat vector bundles 
on $S$ and $X$ by $V_{\chi}^{S}$ and $V_{\chi}^{X}$ respectively.

Under the condition $h^{o}\left(X, V_{x} \otimes \Delta\right)=0$ (i.e., that $V_{x} \otimes \Delta$ has no global holomorphic sections), it turns out that $V_{\chi} \otimes \Delta$ on $X$ admits a certain kernel function (which is called the Cauchy kernel) which is an analogue of $\frac{I_{m}}{z-w}$ for the trivial bundle on the complex plane. The Cauchy kernel is the reproducing kernel for $H_{J(p)}^{2}\left(S, V_{\chi} \otimes \Delta\right)$. In the case of line bundles the Cauchy kernel can be given explicitly in terms of theta functions [27,31]. In Alpay et al. [1] the Cauchy kernel was used to construct for any given holomorphic mapping $z: S \rightarrow D$ an explicit isometric isomorphism between $H_{2, J(p)}\left(S, V_{\chi} \otimes \Delta\right)$ and $H_{2, J}^{M}$ for appropriate $M$ and $J$. In particular this implies that $H_{2, J(p)}\left(S, V_{x} \otimes \Delta\right)$ is indeed non-degenerate (under the condition $h^{0}\left(X, V_{\chi} \otimes \Delta\right)=0$ ) and actually a Kreĭn space.

\section{Statement of the Main Result}

Suppose that we have two finite bordered Riemann surfaces $S_{1}$ and $S_{2}$. Let $F: S_{1} \rightarrow S_{2}$ be an analytic mapping continuous up to the boundary.

Equivalently we may take $F$ to be a complex analytic mapping $F: X_{1}$ $\rightarrow X_{2}$ between the doubles of $S_{1}$ and $S_{2}$ equivariant with respect to the action of the anti-holomorphic involutions, i.e., such that the diagram

$$
\begin{array}{ccc}
X_{1} & \underline{\tau_{1}} & X_{1} \\
F \downarrow & & \downarrow F \\
X_{2} & \underline{\tau_{2}} & X_{2}
\end{array}
$$

is commutative. Notice that $F: S_{1} \rightarrow S_{2}$ is unramified if and only if $F: X_{1}$ $\rightarrow X_{2}$ is unramified.

We identify as usual a complex holomorphic vector bundle on a complex manifold with a locally free sheaf of its analytic sections. It is easily seen that if $V^{X}$ is a complex holomorphic vector bundle of rank $m$ on a complex manifold $X$ and $F$ is a $n$-sheeted unramified covering, then the direct image $V^{Y}=F * V^{X}$ is a complex holomorphic vector bundle of rank $n m$ on $Y$ and the fiber of $V^{Y}$ at a given point of $Y$ is the direct sum of the fibers of $V^{X}$ at the preimages of this point on $X$.

The main statement of this work is the following

Theorem 3.1 Let F: $S_{1} \rightarrow S_{2}$ be a map of finite bordered Riemann surfaces which is a finite $n$-sheeted unramified covering $\left(F ; S_{1}, S_{2}\right)$, and let $J_{1}(\tilde{p})$ be signature matrices for a unitary flat vector bundle $V_{\chi_{1}}$ on $S_{1}$ of rank $m$. Consider the corresponding extension of $V_{\chi_{1}}$ to the double $X_{1}$ of $S_{1}$ satisfying the symmetry condition

$$
\chi_{1}\left(R^{\tau}\right)^{*} G_{1}(R \tilde{p}) \chi_{1}(R)=G_{1}(\tilde{p}),
$$

for all $R \in \pi_{1}\left(X_{1}, p^{\prime}{ }_{0}\right)$ and all $\tilde{p} \in \tilde{X}$. Choose the bundles $\Delta_{1}$ and $\Delta_{2}$ of half-order differentials on $X_{1}$ and $X_{2}$ respectively, such that

a.) the bundles $\Delta_{i}, i=1,2$ are invariant with respect to the corresponding anti-holomorphic involutions, i.e., $\Delta_{i}^{\tau_{i}}=\Delta_{i}$ and the transition functions of $\Delta_{1}$ and $\Delta_{2}$ are symmetric with respect to $\tau_{1}$ and $\tau_{2^{i}}$

b.) the pull-back of $\Delta_{2}$ is equal to $\Delta_{1}$, i.e., $\Delta_{1}=F * \Delta_{2}$.

\section{Then}

1) the direct image $V_{\chi_{1}}^{X_{2}}=F_{*} V_{\chi_{1}}^{X_{1}}$ is a unitary flat holomorpnic vector bundle of rank $n m$ satisfying the symmetry condition

$$
\chi_{2}\left(T^{\tau}\right)^{*} G_{2}(T \tilde{p}) \chi_{2}(T)=G_{2}(\tilde{p}),
$$

for all $T \in \pi_{1}\left(X_{2}, p_{0}\right)$ and all $\tilde{p} \in \tilde{X}$, appropriate matrix function $G_{2}(\tilde{p})$ , and representation $\chi_{2}$ of $\pi_{1}\left(X_{2}, p_{0}\right)$; furthermore $F_{*}\left(V_{\chi_{1}} \otimes \Delta_{1}\right)=V_{\chi_{2}} \otimes \Delta_{2}$;

\section{2) there exists a canonical isometric isomorphism}

$$
\phi_{F}: H_{2, J_{1}(p)}\left(S_{1}, V_{\chi_{1}}, \otimes \Delta_{1}\right) \stackrel{\sim}{\rightarrow} H_{2, J_{2}(p)}\left(S_{2}, V_{\chi_{1}} \otimes \Delta_{2}\right),
$$

between Hardy spaces on $S_{1}$ and $S_{2}$.

Now some remarks are in order. By definition the anti-holomorphic involutions $\tau_{1}$ and $\tau_{2}$ are related by

$$
F \circ \tau_{1}=\tau_{2} \circ F,
$$

and therefore if we have a line bundle $L_{2}$ on $X_{2}$ then its pull-back satisfies

$$
\left(F^{*} L_{2}^{\tau_{2}}\right)=\left(F^{*} L_{2}\right)^{\tau_{1}} \text {. }
$$

We fix $\Delta_{2}$ such that $\Delta_{2}^{\tau}=\Delta_{2}$ and $\Delta_{1}=F^{*} \Delta_{2}$. Then it follows that $\Delta_{1}^{\tau}=\Delta_{1}$. We choose $\Delta_{2}$ such that its transition functions are symmetrical. Then since $F$ is equivariant with respect to the antiholomorphic involution then transition functions of $\Delta_{1}$ are also symmetrical.

The isomorphism of the spaces $H_{2, J_{1}(p)}\left(S_{1}, V_{\chi_{1}} \otimes \Delta_{1}\right)$ and $H_{2, J_{2}(p)}\left(S_{2}, V_{\chi_{2}} \otimes \Delta_{2}\right)$ implies that they are degenerate or nondegenerate simultaneously, i.e., $h^{0}\left(X_{1}, V_{\chi_{1}} \otimes \Delta_{1}\right)=h^{0}\left(X_{2}, V_{\chi_{2}} \otimes \Delta_{2}\right)=0$ which is obvious from the definition of the direct image vector bundle.

We assume that the map $F: S_{1} \rightarrow S_{2}$ is a $n$-sheeted unramified covering $\left(F ; S_{1}, S_{2}\right)$ of the Riemann surface $S_{2}$ by $S_{1}$. On the other hand, a result of Alpay et al. [1] mentioned in Introduction is a construction of an isometric isomorphism between Hardy spaces when $S_{2}=\mathbf{D}$ but $F$ is (usually) ramified (assuming $H_{2, J_{1}(p)\left(S_{1}, V_{\chi_{1}} \otimes \Delta_{1}\right)}$ is not degenerate, i.e., $h^{0}\left(X_{1}, V_{\chi_{1}} \otimes \Delta_{1}\right)=0$. The next natural step would be to consider the case when $S_{2}$ is an arbitrary finite bordered Riemann surface and $F$ is a ramified covering. That will be a point of some further publication.

We have introduced the vector bundle $V_{\chi_{2}}^{X_{2}}$ on the double $X_{2}$ as the direct image of the vector bundle $V_{\chi_{1}}^{X_{1}}$ on $X_{1}$ defining the vector bundle $V_{\chi_{2}}^{s_{2}}$ on $S_{2}$ and the signature matrices $J_{2}(\tilde{p})$. On the other hand one can define the vector bundle $V_{\chi_{2}}^{s_{2}}$ to be the direct image $\mathrm{F} * V_{\chi_{1}}^{s_{1}}$ of the vector bundle $V_{\chi_{1}}^{s_{1}}$ with signature matrices defined naturally in terms of $J_{1}(\tilde{p})$ (as direct sums). Though the main claim of Theorem 3.1 is formulated for finite bordered Riemann surfaces it seems to us that the consideration of the structures involved in its proof is more natural (in the sense of the theory of compact Riemann surfaces) on the doubles. Furthermore, this approach allows us to construct a matrix representation $\chi_{2}$ of the fundamental group $\pi_{1}\left(X_{2}, p_{0}\right)$ given a representation of $\pi_{1}\left(X_{1}, p_{0}^{\prime}\right)$, and the matrix function $G_{2}(\tilde{p})$. We will prove that signature matrices $J_{2}(\tilde{p})$ calculated with the help of the representation $\chi_{2}$ do coincide with the signature matrices constructed directly from the signature matrices $J_{1}(\tilde{p})$. This shows the equivalence of those two approaches. From the use of Cauchy kernels in Alpay et al. [1] it seems however that in the ramified case the approach via the doubles is the only one possible.

Speaking in more abstract terms we deal in Theorem 3.1 with a category which we will denote by $\mathcal{R} \mathcal{H}$. Objects of $\mathcal{R} \mathcal{H}$ are finite bordered Riemann surfaces $S$ together with a unitary flat vector bundle $V_{\chi}$ and signature matrices $J(\tilde{p})$ (or equivalently, compact real Riemann surfaces $X$ of dividing type with a vector bundle $V_{\chi}^{X} \otimes \Delta$ on $X$ and a matrix function $G(\tilde{p})$ satisfying (4) and (5)) such that the space $H_{2, J(p)}\left(S, V_{\chi} \otimes \Delta\right)$ is non degenerate, i.e., $h^{0}\left(X, V_{\chi} \otimes \Delta\right)=0$. A morphism between the objects $\left(X_{1}, V_{\chi_{1}}^{X_{1}} \otimes \Delta_{1}, G_{1}\right)$ 
and $\left(X_{2}, V_{\chi_{2}}^{X_{2}} \otimes \Delta_{2}, G_{2}\right)$ of $\mathcal{R H}$ is an analytic map $F: X_{1} \rightarrow X_{2}$ of Riemann surfaces which is equivariant with respect to anti-holomorphic involutions $\tau_{1}$ and $\tau_{2}$, such that $V_{\chi_{2}}^{X_{2}} \otimes \Delta_{2}=F_{*}\left(V_{\chi_{1}}^{X_{1}} \otimes \Delta_{1}\right)$ (and $G_{2}(\tilde{p})$ is correspondingly induced by $\left.G_{1}(\tilde{p})\right)$.

We conjecture that there exists a covariant functor from the above men-tioned category $\mathcal{R H}$ to the category of Krein spaces and isomorphisms, asso-ciating to $\left(S, V_{\chi} \otimes \Delta, J(\tilde{p})\right)$ the Hardy space $H_{2, J(p)}\left(S, V_{\chi} \otimes \Delta\right)$.

Theorem 3.1 proves the conjecture for a subcategory of $\mathcal{R H}$ whose morphisms are unramified coverings. The isometric isomorphism established in Alpay et al. [1] proves another special case of the conjecture namely for a subcategory whose morphisms are restricted to have the unit disk $\mathbf{D}$ as a range. Somewhat related considerations of categories of functional spaces on Riemann surfaces are contained in Alling et al. [32].

\section{Sections of the Vector Bundle $F_{*}\left(V_{\chi_{1}} \otimes \Delta_{1}\right)$}

In this section we give an explicit construction of a holomorphic section $\hat{f}^{2}$ of the bundle $F_{*}\left(V_{\chi_{1}} \otimes \Delta_{1}\right)$ on $X_{2}$ in terms of a holomorphic section $\hat{f}^{1}$ of $V_{\chi_{1}} \otimes \Delta_{1}$ on $X_{1}$. When $\left(F ; S_{1}, S_{2}\right)$ is an unramified covering the doubles $X_{1}$ and $X_{2}$ possess the common universal covering $\tilde{X}$, i.e., one has a diagram

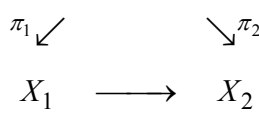

where $\pi_{1}$ and $\pi_{2}$ are the covering maps from $\tilde{X}$ to $X_{1}$ and $X_{2}$ respectively.

Let $U^{\prime} \subset X_{1}$ be an open set in $X_{1}$. Suppose $f^{1} \quad$ is an analytic section of the holomorphic vector bundle $V_{\chi_{1}}$ over $U^{\prime}$, i.e., an analytic $\mathbb{C}^{m}$ valued function on $\pi_{1}^{-1}\left(U^{\prime}\right) \subset \tilde{X}$ satisfying the relation

$$
f^{1}(T \tilde{p})=\chi_{1}(T) f^{1}(\tilde{p}) .
$$

Similarly, a section $\hat{f}^{1}$ of the vector bundle $V_{\chi_{1}} \otimes \Delta$ over $U^{\prime}$ satisfies

$$
\frac{\hat{f}^{1}(T \tilde{p})}{\sqrt{d t_{1}(T \tilde{p})}}=\chi_{1}(T) \frac{\hat{f}^{1}(\tilde{p})}{\sqrt{d t_{1}(\tilde{p})}},
$$

for all $\tilde{p} \in \pi_{1}^{-1}\left(U^{\prime}\right), T \in \pi_{1}\left(X_{1}, p_{0}^{\prime}\right)$, where $t_{1}$ is a local parameter on $X_{1}$ lifted to $\tilde{X}$. The fundamental group $\pi_{1}\left(X_{1}, p_{0}^{\prime}\right), p^{\prime} \in X_{1}$, is a subgroup of $\pi_{1}\left(X_{2}, p_{0}\right)$ of index $n$ (here $p_{0}^{\prime}$ is a preimage of $\left.p_{0} \in X_{2}\right)$. Enumerate fixed representatives $g_{i}, i=1, \ldots, n$ of the left cosets $\left\{\pi_{1}\left(X_{1}, p_{0}^{\prime}\right) g_{i}\right\}$ of the group $\pi_{1}\left(X_{2}, p_{0}\right)$ with respect to its subgroup $\pi_{1}\left(X_{1}, p^{\prime}\right)$. We define a sheaf on $X_{2}$ whose sections over an open set $U \subset X_{2}$ are analytic $\mathbb{C}^{m n}$ valued functions on $\pi_{1}^{-1}(U)$ of the vector form

$$
f^{2}(\tilde{p})=\left[f^{1}\left(g_{i} \tilde{p}\right)\right],
$$

$i=1, \ldots, n$, where $f^{1}$ is a section of the bundle $V_{\chi_{1}}$ over $F^{-1}(U)$,i.e., $f^{1}(\tilde{p})$ is an analytic $\mathbb{C}^{m}$-valued function on $\pi_{1}^{-1}\left(F^{-1}(U)\right)=\pi_{2}^{-1}(U)$ satisfying (11). It easy to see from the definition that this sheaf on $X_{2}$ is isomorphic to the direct image sheaf of a sheaf on $X_{1}$ of analytic sections of $V_{\chi_{1}}$, i.e., (12) defines the sheaf of analytic sections of $F * V_{\chi_{1}}$.

Now let $p \in X_{2}$ and $p_{1}^{\prime}, \ldots, p_{n}^{\prime} \in X_{1}$ be preimages of $p$. Let $t_{2}$ and $t_{1}{ }^{\prime}$ be local parameters near $p$ and $p_{i}^{\prime}, i=1, \ldots, n$ lifted to the common universal covering $\tilde{X}$. Denote by $\varphi_{\mathrm{i}}$ the composition $t_{2}^{-1} \mathrm{o} F \mathrm{o} t_{1, i}$. Then a section $\hat{f}^{2}(\tilde{p})$ of a vector bundle $F_{*}\left(V_{\chi_{1}}^{X_{2}} \otimes \Delta_{1}\right)$ is given by

$$
\hat{f}^{2}(\tilde{p})=\left[\frac{\hat{f}^{1}\left(g_{i} \tilde{p}\right)}{\sqrt{\varphi_{i}^{\prime}\left(g_{i} \tilde{p}\right)} \sqrt{d t_{1, i}\left(g_{i} \tilde{p}\right)}}\right] \sqrt{d t_{2}(\tilde{p})},
$$

where $\hat{f}^{1}(\tilde{p})$ is a section of the vector bundle $V_{\chi_{1}} \otimes \Delta_{1}$ and $t_{1_{1},}, i=1$, $\ldots, n$ are local parameters in the vicinity of $g_{i} \tilde{p}$. Since we have chosen the bundles $\Delta_{1}$ and $\Delta_{2}$ of half-order differentials in (13), the ambiguity in the sign of the square roots of $\varphi^{\prime}\left(g_{i} \tilde{p}\right)$ in (13) is global and since we have assumed that $\Delta_{1}=F * \Delta_{2}$, the expression (13) does not depend on the choice of local parameters.

\section{Representation $\chi_{2}$ of $\pi_{1}\left(X_{2^{2}}, p_{0}\right)$}

In this section we give an explicit formula for a unitary representation $\chi_{2}$ of $2 \pi_{1}\left(X_{2}, p_{0}\right)$ such that $V_{\chi_{2}}=F_{*} V_{\chi_{1}}$. It follows from the previous section that we have to define $\chi_{2}$ so that

$$
f^{2}(T \tilde{p})=\chi_{2}(T) f^{2}(\tilde{p}),
$$

for every $f^{2}$ given by (12). Let $g \in \pi_{1}\left(X_{2}, p_{0}\right)$. Fix a preimage $p^{\prime}{ }_{0} \in X_{1}$ of $p_{0}$. The element $g$ belongs to a coset of the fundamental group $\pi_{1}\left(X_{2}, p_{0}\right)$ with respect to its subgroup $\pi_{1}\left(X_{1}, p_{0}^{\prime}\right)$. Then there exist elements $h \in$ $\pi_{1}\left(X_{1}, p_{0}^{\prime}\right)$ and $g_{\sigma_{g}(i)} \in \pi_{1}\left(X_{2}, p_{0}\right)$ such that

$$
g_{i} g=h g_{\sigma_{g}}(i),
$$

i.e., $g$ defines a permutation $\sigma_{g}$ of the preimages of $p_{0}$. We take this as a definition of $\sigma_{g}$. We define the matrix representation $\chi_{2}$ as follows:

$$
\left[\chi_{2}(g)\right]_{k j}=\chi_{1}\left(g_{k} g g_{\sigma_{g}(k)}^{-1}\right) \delta_{\sigma_{g}(k) j} .
$$

It is immediate that (14) is verified. Taking into account the unitarity of $\chi_{1}(g)$, it can be seen from (15) that the matrices defining the representation of $\pi_{1}\left(X_{2}, p_{0}\right)$ are unitary, i.e.,

$\left[\chi_{2}\left(g_{i}\right) \cdot \chi_{2}\left(g_{i}\right)^{*}\right]_{k j}=\delta_{k j}$.

Now we check that (15) provides a representation $\pi_{1}\left(X_{2}, p_{0}\right)$, i.e.,

$$
\chi_{2}(g \tilde{g})=\chi_{2}(g) \chi_{2}(\tilde{g}) \text {, }
$$

for all $g, \tilde{g} \in \pi_{1}\left(X_{2}, p_{0}\right)$. Proving this we used the fact that $\chi_{1}$ is homomorphism and $\sigma_{g}$ is an anti-homomorphism, i.e.,

$$
\sigma_{g^{\prime}}\left(\sigma_{g^{\prime \prime}}(k)\right)=\sigma_{g^{\prime \prime} g^{\prime}}(k),
$$

which can be easily verified. In general, the matrix $\chi_{2}$ is given by the formula

$$
\left[\chi_{2}(g)\right]_{k j}=\chi_{1}\left(g_{k} g g_{j}^{-1}\right) \delta_{k+i-j-1,0},
$$

where $g$ belongs to $i$-th coset.

\section{Construction of Pairing and Inner Product}

Suppose that $H^{1}=V_{\chi_{1}} \otimes \Delta_{1}$ is such that there exists a non-degenerate bilinear pairing $H^{1} \times\left(H^{1}\right)^{\tau} \longrightarrow K_{X_{1}}$ which is parahermitian, i.e.,

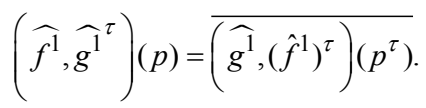

We assume that the line bundle $\Delta_{1}$ is such that $\Delta_{1}^{\tau_{1}} \cong \Delta_{1}$ and the transition functions of $\Delta$ are symmetric with respect to $\tau_{1}$. Then we have a parahermitian non-degenerate bilinear pairing $V_{\chi_{1}} \otimes V_{\chi_{1}}^{\tau} \longrightarrow \mathrm{O}_{X_{1}}$ 
and the matrix function $G_{1}(\tilde{p})$ satisfying (4) and (5). One can define a bilinear non-degenerate pairing $H^{2} \times\left(H^{2}\right)^{\tau} \longrightarrow K_{X_{2}}$ where $H^{2}=F * H^{1}=V_{\chi_{2}} \otimes \Delta_{2}$, introducing an everywhere nonsingular holomorphic $m n \times m n$ matrix-valued function $G_{2}$ on the universal covering $\tilde{X}$ of $X_{1}$ and $X_{2}$. The matrix $G_{2}(\tilde{p})$ should have the property

$$
G_{2}\left(\tilde{p}^{\tau}\right)^{*}=G_{2}(\tilde{p})
$$

and satisfy the symmetry condition, $T \in \pi_{1}\left(X_{2}, p_{0}\right)$,

$$
\chi_{2}\left(T^{\tau}\right)^{*} G_{2}(T \tilde{p}) \chi_{2}(T)=G_{2}(\tilde{p}) .
$$

Then the pairing is given by

$$
\left(\widehat{f^{2}}, \widehat{g^{2}}\right)(\tilde{p})=\widehat{g^{2}}\left(\tilde{p}^{\tau}\right)^{*} G_{2}(\tilde{p}) \widehat{f^{2}}(\tilde{p})
$$

Taking into account the explicit form (15) of $\chi_{2}(g)$ one can check that the following expression for $G_{2}(\tilde{p})$ does satisfies (17)

$$
\left[G_{2}(\tilde{p})\right]_{k j}=G_{1}\left(g_{k}^{\tau} \tilde{p}\right) \chi_{1}\left(h_{k}\right) \delta_{v(k), j},
$$

where $v(k)$ is defined as follows. Consider the action of $\tau$ on an element $g \in \pi_{1}\left(X_{2}, p_{0}\right)$. By definition we have $g^{\tau}=\tau g \tau^{-1}$. For any $g^{k}$ that belongs to $k$-th coset of $\pi_{1}\left(X_{2}, p\right)$ with respect to $\pi_{1}\left(X_{1}, p^{\prime}{ }_{0}\right)$ there exist $h_{k} \in \pi_{1}\left(X_{1}\right.$, $\left.p^{\prime}{ }_{0}\right)$ and $g_{v(k)} \in \pi_{1}\left(X_{2}, p\right)$ such that

$$
g_{k}^{\tau}=h_{k} g_{v(k)} \text {. }
$$

We define $v(k)$ by (19). One can check directly that (18) does satisfy conditions (16) and (17).

We saw in Introduction how to define an indefinite inner product (2), (9) on the Hardy space $H_{2, J_{0}, \ldots, J_{k-1}}\left(S, V_{\chi} \otimes \Delta\right)$ using signature matrices $J_{0}, \ldots, J_{k-1}$. Suppose that we have such an inner product on $H_{2, J_{1,0}, \ldots, J_{1, k-1}}\left(S_{1}, V_{\chi_{1}} \otimes \Delta_{1}\right)$

$$
\left[\widehat{f^{1}}, \widehat{g^{1}}\right]_{H_{2, J_{1}, 0, \ldots, J_{1, k-1}}\left(S_{1}, V_{\chi_{1}} \otimes \Delta_{1}\right)}=\sum_{i=0}^{k-1} \int_{X_{1, i}} \widehat{g^{1}}(\tilde{p})^{*} J_{1, i} \widehat{f^{1}}(\tilde{p}) .
$$

Then we define an indefinite inner product on $H_{2, J_{2,0}, \ldots, J_{2, k-1}}\left(S, V_{\chi_{2}} \otimes \Delta_{2}\right)$

$$
\left[\widehat{f^{2}}, \widehat{g^{2}}\right]_{H_{2, J_{2,0}, \ldots, J_{2, k-1}}\left(S, V_{\chi_{2}} \otimes \Delta_{2}\right)}=\int_{X_{2, i}} \widehat{g^{2}}\left(\tilde{p}^{\tau_{2}}\right)^{*} G_{2}(\tilde{p}) \widehat{f^{2}}(\tilde{p}) .
$$

By the same reasons as in Introduction we can rewrite (20) as

$$
\left[\widehat{f^{2}}, \widehat{g^{2}}\right]_{H_{2, J_{2,0}, \ldots, J_{2, k-1}}\left(S, V_{\chi_{2}} \otimes \Delta_{2}\right)}=\int_{X_{2, i}} \widehat{g^{2}}(\tilde{p})^{*} J_{2}(\tilde{p}) \widehat{f^{2}}(\tilde{p}),
$$

Where

$$
J_{2}(\tilde{p})=\chi_{2}\left(T_{\tilde{p}}\right)^{*} G_{2}(\tilde{p}),
$$

and introduce the matrices

$$
J_{2,0}=G_{2}, \quad J_{2, i}=\chi_{2}\left(B_{2,1}\right)^{*} G_{2},
$$

where $B_{2,1} \in \pi\left(X_{2}, p\right)$ (refer Appendix). As in Alpay et al. [1] the extension of the bundle $V_{\chi_{2}}^{S_{2}}$ on the Riemann surface $S_{2}$ to the double $X_{2}$ depends on the choice of the signature matrices $J_{2,0}, J_{2, \mathrm{k}-1}$ given by (22) and which satisfies the symmetry condition (8). On the other hand, one can define the signature matrix $J_{2}(\tilde{p})$ using the signature matrix $J_{1}(\tilde{p})$. One should have

$$
\chi_{2}(T)^{*} J_{2}(T \tilde{p}) \chi_{2}(T)=J_{2}(\tilde{p})
$$

for all $T \in \pi_{1}\left(X_{2}, p\right)$ and

$$
J_{2}(\tilde{p})^{*}=J_{2}(\tilde{p}),
$$

for all $\tilde{p} \in \tilde{X}$ over $p \in X_{2, f}$ The matrix $J_{2}(\tilde{p})$ in the form

$$
\left[J_{2}(\tilde{p})\right]_{k j}=J_{1}\left(g_{k} \tilde{p}\right) \delta_{k j},
$$

satisfies (23) and (24). Then we check the commutativity of the diagram

$$
\begin{array}{ccc}
V_{\chi_{1}}^{S_{1}} & \stackrel{e x t, J_{1}}{\longrightarrow} & V_{\chi_{1}}^{X_{1}} \\
F_{*}^{S} \downarrow & & \downarrow F_{*}^{X} \\
V_{\chi_{2}}^{S_{2}} & \stackrel{e x t, J_{2}}{\longrightarrow} & V_{\chi_{2}}^{X_{2}}
\end{array}
$$

Where ext, $J_{i}$ means the extension of the vector bundle $V_{\chi_{i}}^{s_{i}}$ on $S_{i}$ to the double $X_{\mathrm{i}}$. I.e., we will show that the matrix $J_{2}(\tilde{p})$ defined by (22) coincides with (25). It easy to check that

$$
T_{R \tilde{p}} R=R^{\tau} T_{\tilde{p}}
$$

for all $R \in \pi_{1}\left(X_{2}, p\right), p \in X_{2, f}$ and $T_{\tilde{p}} \in \pi\left(X_{2}, p\right)$ such that $\tilde{p}^{\tau}=T_{\tilde{p}} \tilde{p}$ where $\tilde{p}$ lies over $p$. Using that we arrive at

$$
\left[J_{2}(\tilde{p})\right]_{k j}=\chi_{2}\left(T_{\tilde{p}}\right)^{*} G_{2}(\tilde{p})=J_{1}\left(g_{k} \tilde{p}\right) \delta_{k j} .
$$

\section{Proof of the Isometricity}

We have constructed explicitly a section $\widehat{f^{2}}(13)$ of the bundle $V_{\chi_{2}} \otimes \Delta_{2}$ in terms of a section $\widehat{f^{1}}$ of the bundle $V_{\chi_{1}} \otimes \Delta_{1}$. Now we will prove that the map $\widehat{f^{1}} \mapsto \widehat{f^{2}}$ is an isometric isomorphism of the space $H_{2, J_{1}(p)}\left(S_{1}, V_{\chi_{1}} \otimes \Delta_{1}\right)$ on the space $H_{2, J_{2}(p)}\left(S_{2}, V_{\chi_{2}} \otimes \Delta_{2}\right)$. First let us show that $\widehat{f^{1}} \in H_{2, J_{1}(p)}\left(S_{1}, V_{\chi_{1}} \otimes \Delta_{1}\right)$ if and only if $\widehat{f^{2}} \in H_{2, J_{2}(p)}\left(S_{2}, V_{\chi_{2}} \otimes \Delta_{2}\right)$.

Suppose $\widehat{f^{1}}$ is a section of the bundle $V_{\chi_{1}} \otimes \Delta_{1}$ and $\widehat{f^{1}} \in H_{2, J_{1}(p)}\left(S_{1}, V_{\chi_{1}} \otimes \Delta_{1}\right)$. That means that $\widehat{f}^{1} \in H_{2},\left(S_{1}, V_{\chi_{1}} \otimes \Delta_{1}\right)$, i.e., $\widehat{f^{1}}$ is an analytic in $X_{1}$

$$
\begin{aligned}
& \text { And } \\
& \sup _{1-\epsilon<r<1} \sum_{i=0}^{k-1} \int_{\mathcal{X}_{1, i}(r)} \widehat{f^{1}}(p)^{*} \widehat{f^{1}}(p)<\infty,
\end{aligned}
$$

for some $\epsilon$. Here $\mathcal{X}_{1, i}(r)$ are smooth simple curves in $X_{1}$ approximating the $i$-th boundary of the $X_{1}$. The space $H_{2, J_{1}(p)}\left(S_{1}, V_{\chi_{1}} \otimes \Delta_{1}\right)$ is the space $H_{2}\left(S_{1}, V_{\chi_{1}} \otimes \Delta_{1}\right)$ endowed with the indefinite inner product (2)

$$
\widehat{\left[f^{1}, \widehat{g^{1}}\right]_{H_{2, J_{1,0, \ldots,}, J_{1, k-1}}}\left(S_{1}, V_{\chi 1} \otimes \Delta_{1}\right)}=\sum_{i=0}^{k-1} \int_{\tilde{\chi}_{1, i}} \widehat{g^{1}}(\tilde{p})^{*} J_{1, i} \widehat{f^{1}}(\tilde{p}) \text {. }
$$

Let $\mathcal{X}_{2, i}$ be a boundary component of $X_{2}$ and $\mathcal{X}_{1, i}, j=1, \ldots, n_{i}$ be corresponding preimages on $X_{1}$. The boundary uniformizer $z_{1}$ near the boundary component is such that $z_{1} p_{0}^{\prime}=z_{2}{ }^{\circ} F p_{0}^{\prime}$. Then the approximating curves $\mathcal{X}_{2, i}(r)$ are mapped to the approximating curves $\mathcal{X}_{1, i_{j}}(r), j=1, \ldots, n_{i}$. Due to the construction given by the formula (13) we see that $\widehat{f^{2}}$ is an analytic and

$$
\begin{aligned}
& \sum_{i=0}^{k_{2}-1} \int_{X_{2, i}(r)} \widehat{f^{2}}(p)^{*} \widehat{f^{2}}(p)=\sum_{i=0}^{k_{2}-1} \sum_{j=1}^{n_{i}} \int_{\tilde{\chi}_{1, i_{j}}} \widehat{f^{1}}(\tilde{p})^{*} \widehat{f^{1}}(\tilde{p}) \\
& =\sum_{i=0}^{k_{1}-1} \int_{X_{1, i}} \widehat{f^{1}}(p)^{*} \widehat{f^{1}}(p)<\infty .
\end{aligned}
$$


Citation: Zuevsky A (2015) Hardy Spaces on Compact Riemann Surfaces with Boundary. J Generalized Lie Theory Appl S1: 005. doi:10.4172/17364337.S1-005

The summation in (26) with upper limits $n_{i}$ is performed over the components $\mathcal{X}_{1, i}, j=1, \ldots, n_{i}$ that are preimages of $\mathcal{X}_{2, i}$. Thus we infer that $\widehat{f^{2}}$ belongs to the space $H_{2}\left(S_{2}, V_{\chi_{2}} \otimes \Delta_{2}\right)$. In the previous section we have introduced an indefinite inner product in the space $H_{2}\left(S_{2}, V_{\chi_{2}} \otimes \Delta_{2}\right)$. Thus we see that a section $\widehat{f^{2}}$ of xconstructed by the formula (13) belongs to the space $H_{2, J_{2}(p)}\left(S_{2}, V_{\chi_{2}} \otimes \Delta_{2}\right)$.

Finally, it remains to show that the inner product (21) is isometric, i.e., that

$$
\begin{aligned}
& {\left[\widehat{f^{2}}(\tilde{p}), \widehat{h^{2}}(\tilde{p})\right]_{H_{2, J_{2,0, \ldots,} J_{2, k-1}}\left(S_{2}, V_{\chi_{2}} \otimes \Delta_{2}\right)}} \\
& =\left[\widehat{f^{1}}(\tilde{p}), \widehat{h}^{1}(\tilde{p})\right]_{H_{2, J_{1,0, \ldots, \ldots, k-1}}\left(S_{1}, V_{\chi_{1}} \otimes \Delta_{1}\right)},
\end{aligned}
$$

where $\widehat{f^{1}}, \widehat{h^{1}}$ and $\widehat{f^{2}}, \widehat{h^{2}}$ are sections of the vector bundles $V_{\chi_{1}} \otimes \Delta_{1}$ and $V_{\chi_{2}} \otimes \Delta_{2}$ respectively. Indeed, consider the inner product of two sections of the bundle $V_{\chi_{2}} \otimes \Delta_{2}$

$$
\begin{aligned}
& {\left[\widehat{f^{2}}(\tilde{p}), \widehat{h^{2}}(\tilde{p})\right]_{H_{2, J_{2,0, \ldots,}, J_{2, k-1}}\left(S_{2}, V_{\chi_{2}} \otimes \Delta_{2}\right)}=\sum_{l=0}^{k_{2}-1} \int_{\tilde{\chi}_{2, l}} \widehat{f^{2}}(\tilde{p}) * J_{2, l} \widehat{h^{2}}(\tilde{p})} \\
& =\int_{\tilde{\chi}_{2, f}} \widehat{f^{2}}\left(\tilde{p}^{\tau}\right) * G_{2}(\tilde{p}) \widehat{h^{2}}(\tilde{p}) \\
& =\int_{\tilde{\chi}_{2, f}} \sum_{i, j=1}^{n}{\widehat{f^{1}}}^{1}\left(\left(g_{i}^{\tau} \tilde{p}\right)^{\tau}\right)^{*} G_{1}\left(g_{i}^{\tau} \tilde{p}\right) \widehat{h}^{1}\left(g_{i}^{\tau} \tilde{p}\right) \frac{d t_{2}(\tilde{p})}{\varphi_{i}^{\prime}\left(g_{i} \tilde{p}\right) d t_{1, i}\left(g_{i} \tilde{p}\right)} \text {. }
\end{aligned}
$$

By the same argument that were used in the formulae (26) the last integral is equal to

$$
\begin{aligned}
& \int_{\tilde{\chi}_{1, f}} \widehat{f^{1}}\left(\tilde{p}^{\tau}\right) * G_{1}(\tilde{p}) \widehat{h^{1}}(\tilde{p})=\sum_{l=0}^{k_{1}-1} \int_{\tilde{\chi}_{1, l}} \widehat{f^{1}}(\tilde{p}) * J_{l, l} \widehat{h^{1}}(\tilde{p}) \\
& =\left[\widehat{f^{1}}(\tilde{p}), \widehat{h^{1}}(\tilde{p})\right]_{H_{2, J_{1}, 0, \ldots, J_{1, k-1}}\left(S_{1}, V_{\chi_{1}} \otimes \Delta_{1}\right)},
\end{aligned}
$$

where we use the invariance of sections of $\Delta_{1}$ with respect to deck transformations and the symmetry of the their transition functions. Hence we see that (27) holds. That completes the proof of the isometricity.

\section{Appendix: Fundamental groups of $S$ and Double $X$}

Let us describe explicitly [1] the action of $\tau$ on the generators of $\pi_{1}\left(X, p_{0}\right)$. Choose points $p_{i} \in \mathcal{X}, i=0, \ldots, k-1$, and let $C_{\mathrm{i}}$ be a path on $S$ linking $p_{0}$ to $p_{i}$. Then $\pi_{1}\left(S, p_{0}\right)$ is generated by

$$
A_{0}, A_{1}, \ldots, A_{k-1}, A_{1}^{\prime}, B_{1}^{\prime}, \ldots, A_{S}^{\prime}, B_{S}^{\prime} \text {, }
$$

where $A_{0}=\mathcal{X}_{0}, A_{j}=C_{j}^{-1} \mathcal{X}_{j} C_{j}$ for $j=1, \ldots, k-1$ and $A_{i}^{\prime}, B_{i}^{\prime}, i=1, \ldots, s$, represent a canonical homology basis on $S$ with the intersection matrix $\left(\begin{array}{cc}0 & I \\ -I & 0\end{array}\right)$. The generators of $\pi_{1}\left(S, p_{0}\right)$ satisfy a single relation

$$
\prod_{i=1}^{s} A_{i}^{\prime} B_{i}^{\prime} A_{i}^{\prime-1} B_{i}^{\prime-1} \prod_{k-1}^{0} A_{i}=1 \text {. }
$$

Now consider the fundamental group $\pi_{1}\left(X, p_{0}\right)$. It is generated by $A_{1}, B_{1}, \ldots, A_{k-1}, B_{k-1}, A_{1}^{\prime}, B_{1}^{\prime}, \ldots, A_{s}^{\prime}, B_{s}^{\prime}, A_{1}^{\prime \prime}, B_{1}^{\prime \prime}, \ldots, A_{s}^{\prime \prime}, B_{s}^{\prime \prime}$.
The generators $A_{j}, A_{i}^{\prime}, B_{i}^{\prime}$ are the same as in (28)

$B_{j}=\left(C_{j}^{\tau}\right)^{-1} C_{j}$,

for $j=1, \ldots, \mathrm{k}-1$ and

$A_{i}^{\prime \prime}=B_{i}^{\prime}{ }^{\tau}$,

$B_{i}^{\prime \prime}=A_{i}^{\prime} \tau$.

The generators of $\pi_{1}\left(X, p_{0}\right)$ satisfy a single relation by Natanzon et al. [33-41]

$\prod_{i=s}^{1} A_{i}^{\prime \prime} B_{i}^{\prime \prime} A_{i}^{\prime \prime}-1 B_{i}^{\prime \prime}-1 \prod_{i=1}^{s} A_{i}^{\prime} B_{i}^{\prime} A_{i}^{\prime-1} B_{i}^{\prime}{ }^{-1} \prod_{j=k-1}^{1} A_{j} \prod_{j=1}^{k-1} B_{j} A_{j}^{-1} B_{j}^{-1}=1$.

Note that

$$
\begin{aligned}
B_{j}^{\tau} & =B_{j}^{-1} \\
A_{j}^{\tau} & =B_{j} A_{j} B_{j}^{-1} .
\end{aligned}
$$

\section{References}

1. Alpay D, Vinnikov V (2000) Indefinite hardy spaces on finite bordered riemann surfaces. J Funct Anal 172: 221-248.

2. Abrahamse MB, Douglas RG (1976) A class of subnormal operators related to multiply connected domains. Adv in Math 19: 106-148.

3. Rudin W (1955) Analytic functions of class Hp. Trans Amer Math Soc 78: 46-66.

4. Sarason D (1965) The Hp spaces of an annulus. (1stedn), Mem Amer Math Soc.

5. Vinnikov V (1998) Commuting operators and function theory on a Riemann surface. Math Sci Res Inst Publ.

6. Voichik M (1964) Ideals and invariant subspaces of analytic functions. Trans Amer Math Soc 111: 493-512.

7. Mason G, Tuite MP, Zuevsky A (2008) Torus n-Point Functions for R-graded Vertex Operator Superalgebras and Continuous Fermion Orb-ifolds. Commun Math Phys 283: 305-342.

8. Tuite MP, Zuevsky A (2011) The Szeg”o Kernel on a Sewn Riemann Surface. Commun Math Phys 306: 617-645.

9. Tuite MP, Zuevsky A (2011) The genus two partition and correlation functions for free fermionic vertex operator superalgebras. Commun Math Phys 306: 419-447.

10. Fay JD (1992) Kernel functions, analytic torsion, and moduli spaces. Memoirs of the Amer Math Soc.

11. Yamada A (1980) Precise variational formulas for abelian differentials. Koda Math J 3: 114-143.

12. Ball JA, Vinnikov V (2001) Hardy spaces on a finite bordered Riemann surface, multivariable operator model theory and Fourier analysis along a unimodular curve. In: Systems, approximation, singular integral operators, and related topics. Oper Theory Adv Appl 129: 37-56.

13. Kravitsky N (1996) Rational operator functions and Bezoutian operator vessels. Integral Equations Operator Theory 26: 60-80.

14. Hoffman K (1962) Banach spaces of analytic functions. Prentice-Hall, Englewood Cliffs, NJ

15. Duren PL (1970) Theory of Hp spaces. Academic Press, New York.

16. Garnett JB (2007) Bounded analytic functions. Academic press, San Diego.

17. Dym H (1989) J-Contractive matrix functions, reproducing kernel spaces and interpolation. CBMS Lecture Notes, Rhodes Island.

18. Birkhuser, Basel, de Branges L (1972) Espaces hilbertiens de fonctions enti eres. Masson, Paris.

19. Bognar (1974) Indefinite inner product spaces. Springer-Verlag, Berlin.

20. lohvidov IS, Kre ın MG, Langer H (1982) Introduction to the spectral theory of operators in spaces with an indefinite metric. Akademie-Verlag, Berlin. 
Citation: Zuevsky A (2015) Hardy Spaces on Compact Riemann Surfaces with Boundary. J Generalized Lie Theory Appl S1: 005. doi:10.4172/17364337.S1-005

Page 8 of 8

21. Ya Azizov T, lohvidov IS (1986) Foundations of the theory of linear operators in spaces with indefinite metric. Nauka, Moscow, (in Russian). English translation: Linear operators in spaces with an indefinite metric. John Wiley, New-York.

22. Ball JA, Vinnikov $V(1996)$ Zero-pole interpolation for meromorphic matrix functions on an algebraic curve and transfer functions of 2D systems. Acta Appl Math 45: 239-316.

23. Vinnikov $V$ (1989) Complete description of determinantal representations of smooth irreducible curves. Linear Algebra Appl 125: 103-140.

24. Vinnikov $\vee$ Commuting nonselfadjoint operators and algebraic curves. Operator Theory and Complex Analysis, Operator Theory and Complex Analysis, Birkhäuser Basel Publishers, Germany.

25. Vinnikov $V$ (1994) 2D systems and realization of bundle mappings on com-pact Riemann surfaces. In U Helmke, R Mennicken and JSaurer, editors, systems and networks: Mathematical theory and applications of Math Res. Akademie Verlag, Berlin 909-912.

26. Ball JA, Vinnikov $V$ (1999) Zero-pole interpolation for matrix meromorphic functions on a compact Riemann surface and a matrix Fay trisecant identity. Amer J Math 121: 841-888.

27. Fay JD (1973) Theta functions on Riemann surfaces. Lecture Notes in Math, Springer-Verlag, New York.

28. Seshadri CS (1982) Fibr'es vectoriels sur les courbes alg'ebriques. Societe' math'ematique de France, Paris.

29. Drezet JM, Narasimhan MS (1989) Groupe de Picard des varetes de mod-ules de fibres semi-stables sur les courbes algebriques. Invent Math 97: 53-94.

30. Vinnikov $\vee(1993)$ Self adjoint determinantal representations of real plane curves. Math Ann 296: 453-479.

31. Ball JA, Clancey K (1996) Reproducing kernels for Hardy spaces on multi-ply connected domains. Integral Equation Operator Theory 25: 35-37.

32. Alling NL (1965) Extensions of meromorphic function rings over non-compact Riemann surfaces. I Math Z 89: 273-299.

33. Natanzon SM (1980) Moduli spaces of real curves. Trans Moscow Math Soc 37: 233-272.

34. Farkas HM, Kra I (1991) Riemann surfaces. (2ndedn), Springer-Verlag.

35. Forster O (1981) Lectures on Riemann surfaces. Springer-Verlag, New York.

36. Griffits P, Harris J (2011) Principles of algebraic geometry. Wiley-Inter science Publication

37. Gunning RC (1966) Lectures on Riemann surfaces. Mathematical notes, Princeton University press.

38. Hartshorne R (1977) Algebraic geometry. Springer-Verlag, New York.

39. Mumford D (1984) Tata lectures on theta. Birkh"auser, Boston.

40. Narasimhan R (1992) Compact Riemann surfaces. Birkh“auser, Verlag.

41. Spanier EH (1966) Algebraic topology. Springer-Verlag, New York.
This article was originally published in a special issue, Algebra, Combinatorics and Dynamics handled by Editor. Dr. Natalia lyudu, Researcher School of Mathematics, The University of Edinburgh, UK 